\title{
Delimitação da aptidão agroclimática para videira sob irrigação no Nordeste Brasileiro ${ }^{1}$
}

\author{
Antônio H. de C. Teixeira ${ }^{2}$, Jorge Tonietto ${ }^{3}$, G iuliano E. Pereira ${ }^{3} \&$ Francislene Angelotti ${ }^{2}$
}

\section{RESU MO}

Dados climáticos médios foram usados em conjunto com modelos simples de regressão que relacionam o coeficiente de cultura $(\mathrm{KC})$, a evapotranspiração de referência (ETO) e os graus dias acumulados (GD ${ }_{\text {ac }}$ ) para quantificar o requerimento hídrico de videiras (RHV) para mesa e para vinho no N ordeste brasileiro. O RHV juntamente com dados de precipitação permitiu o desenvolvimento de um índice hídrico para videiras (IHV), o qual foi aplicado com dados médios de temperatura do ar para os ciclos produtivos na caracterização de diferentes graus de aptidão sob irrigação, variando-se as datas de poda. Para produção de uvas de mesa constatou-se que não há limitação térmica na região, entretanto maiores teores de açúcar, bem como maior produção, podem ser obtidos nos locais com valores de temperatura do ar mais elevados. Com relação a videiras para vinho, ocorre alguma limitação térmica para a elaboração de vinhos de boa qualidade, dependendo da data de poda, com altos teores de álcool e baixa acidez obtidos nas condições de elevados valores de temperatura do ar. A melhor época de poda para videiras de mesa é de julho a setembro, destacando-se as áreas ao oeste dos estados da Bahia, Pernambuco, Paraíba e quase todas as áreas dos estados do Rio Grande do Norte, Ceará, Piauí e Maranhão. Considerando-se videiras para vinho o período com melhor aptidão climática é quando a poda é realizada entre maio e junho, destacando-se os estados da Bahia, Pernambuco, Paraíba, Alagoas, e Sergipe, e ainda a parte sudoeste do Estado do Maranhão.

Palavras-chaves: evapotranspiração, coeficiente de cultura, graus-dias, índices bioclimáticos

\section{Delimitation of Agro-climatic aptitude for vine crop under irrigation in the Northeast Brazil}

\begin{abstract}
Long term weather data were used together with simple regression models involving crop coefficient (KC), reference evapotranspiration (ETO) and accumulated degree days (DD ) to quantify the vineyard water requirement (VWR) of table and wine grapes in the Brazilian N ortheast. VWR joined with precipitation, allowed the development of a vineyard water indicator (VWI), which was applied together with average values of air temperature for a growing season to characterize the agro-climatic aptitude. For table grapes it was observed that the region has no thermal limitation, however higher both sugar contents and grape yield would be obtained in the areas with higher values of air temperature. In relation to wine grape, it was observed that there are some thermal limitations for the wine quality, depending on the pruning date, being higher the alcohol content and lower acidity, in wines elaborated under conditions with higher values of air temperature. In relation to table grapes, the best pruning dates are between June and September, being highlighted the west areas of Bahia, Pernambuco, Paraíba and almost all areas of Rio Grande do N orte, Ceará, Piauí and Maranhão. Considering wine grapes, the best agroclimatic aptitude is for pruning dates from May to June, with highlights for the states of Bahia, Pernambuco, Paraíba, Alagoas and Sergipe, and a southeast area of M aranhão state.
\end{abstract}

Key words: evapotranspiration, crop coefficient, degree days, bioclimatic indices

\footnotetext{
${ }^{1}$ Trabalho apresentado durante a II Reunião Sulamericana para Manejo e Sustentabilidade da Irrigação em Regiões Áridas e Semiáridas, Cruz das Almas, BA - 03 a 07 de abril de 2011

2 Embrapa Semiárido. BR 428, Km 152, Zona Rural, CP 23, CEP 56302-970, Petrolina, PE. Fone: (87) 3866-3600. E-mail: heribert@cpatsa.embrapa.br; fran.angelotti@cpatsa.embrapa.br

Embrapa Uva e Vinho. Rua Livramento, 515, CP 130, CEP 95700-000, Bento Gonçalves, RS. Fones: (54) 3455-8045; (87) 3386-2171. E-mail: tonietto@cnpuv.embrapa.br; gpereira@cpatsa.embrapa.br
} 


\section{INTRODUÇÃO}

A distribuição de parreirais no mundo é condicionada pela radiação solar, temperatura do ar, umidade atmosférica e umidade do solo, que determinam a atividade fotossintética e a evapotranspiração (Teixeira, 2009). A faixa ótima para a fotossíntese ocorre entre 25 e $30^{\circ} \mathrm{C}$. Acima de $30^{\circ} \mathrm{C}$, o peso e o tamanho das uvas são reduzidos, decrescendo os processos metabólicos sob condições próximas de $45{ }^{\circ} \mathrm{C}$. As reações fotossintéticas são menos intensas com valores de temperatura do ar inferiores a $20^{\circ} \mathrm{C}$ (Costacurta \& Roselli, 1980; Coombe, 1987).

A temperatura do ar afeta a fisiologia da videira e a qualidade das uvas, com a concentração de açúcar aumentando e a de ácido diminuindo simultaneamente quando os parreirais são cultivados sob condições térmicas elevadas, influenciando a qualidade das uvas (Teixeira, 2009; Keller, 2010). Os vinhos elaborados nestas condições apresentam maiores teores de álcool, baixa acidez e valores de $\mathrm{pH}$ elevados, afetando negativamente a intensidade e qualidade do aroma, cor e longevidade (Ollat et al., 2002; Orduña, 2010).

O efeito mais considerável da temperatura do ar na qualidade do vinho é na acidez total. Enquanto que o principal ácido das uvas, o ácido tartárico, é relativamente estável com relação aos efeitos térmicos, na fase de maturação os níveis de ácido málico decrescem com valores elevados de temperatura do ar (Tarara et al., 2008). Nestas condições, podem ocorrer problemas de equilíbrio e conservação, com a presença de taninos imaturos, podendo os vinhos elaborados estarem sujeitos a degradações causadas por microorganismos (Ribereau-Gayon et. al, 2004a,b).

Elevados valores de temperatura do ar (acima de $30{ }^{\circ} \mathrm{C}$ ) aumentam a concentração de sólidos suspensos, mas os altos valores de brix $\left(24-25^{\circ}\right)$ podem ser devidos ao aumento das taxas de evapotranspiração (Keller, 2010). A temperatura do ar atua nas taxas evapotranspiratórias, devido ao fato de que a atmosfera aquecida próxima às plantas transfere energia aumentando os fluxos hídricos para a atmosfera.

Para os processos de fotossíntese e evapotranspiração da cultura da videira, a radiação solar é a principal fonte de energia. Além da área foliar, o tipo do sistema de condução e o manejo cultural influenciam estes processos (Teixeira et al., 2007). As diferenças de pressão do vapor entre a superfície cultivada e o ar vizinho é outro fator importante para as taxas evapotranspiratórias. A velocidade do vento atua no processo de evapotranspiração, porque este processo depende da turbulência do ar, a qual é afetada pela arquitetura das plantas (Teixeira et al., 2008).

Climas com baixos índices pluviométricos são mais indicados para o cultivo comercial da videira, embora as variedades para mesa sejam mais sensíveis ao excesso de chuvas, as quais causam danos diretos nas uvas e o consequente aumento da umidade do ar eleva o risco de doenças. Entretanto, climas secos acarretam em umidade do solo insuficiente trazendo a necessidade da irrigação baseada nos requerimentos hídricos dos parreirais (Teixeira, 2009).

A evapotranspiração em parreirais tem sido obtida através de lisímetros (Williams et al., 2003; Williams \& Ayars, 2005), técnicas das correlações turbulentas (Ortega-Farias et al., 2007) e pelo método da razão de Bowen (Rana et al., 2004; Yunusa et al., 2004, Azevedo et al., 2007, Teixeira et al., 2007). Os resultados revelam uma variação significante nos requerimentos de água, com os métodos pontuais falhando na estimativa das variáveis hídricas em larga escala.

A extrapolação de medições pontuais em parreirais pode ser realizada com a utilização de ferramentas como imagens de satélites e Sistemas de Informações Geográficas - SIG (Teixeira, 2009). Moller et al. (2007) demonstraram a eficiência do uso de imagens com alta resolução na faixa do visível e termal na quantificação do requerimento hídrico de parreirais israelenses. Teixeira et al. (2009b) aplicaram imagens Landsat em conjunto com SIG na determinação da produtividade da água em cultivos comerciais de videira para mesa e para vinho na região semiárida do Nordeste do Brasil.

Para a delimitação da aptidão agroclimática, almejando boas produções de uvas e de vinho, podem-se aplicar índices que considerem as condições térmicas e hídricas usando SIG e dados climáticos históricos. Do ponto de vista hídrico, o parâmetro de entrada para o zoneamento é a precipitação, enquanto que a variável da saída é a evapotranspiração (Teixeira et al., 2002).

No atual estudo a evapotranspiração em ótimas condições de umidade (evapotranspiração potencial) é considerada como o requerimento hídrico dos parreirais. Do ponto de vista térmico, os valores médios de temperatura do ar para os ciclos produtivos são usados na qualificação das uvas e do vinho produzidos variando-se a época de poda no Nordeste brasileiro.

O objetivo deste trabalho foi o desenvolvimento e aplicação de índices bioclimáticos em escala regional nesta região, considerando-se diferentes épocas de poda, na delimitação de áreas com variação nos graus de aptidão climática para cultivo comercial da videira irrigada para mesa e para vinho, gerando subsídios para expansão racional de parreirais com menor risco de agressão ambiental e maior possibilidade de sucesso.

\section{Material e MÉTODOS}

Na Figura 1 pode-se observar a Região Nordeste do Brasil com as divisões estaduais e as estações agrometeorológicas convencionais e automáticas utilizadas.

Para o zoneamento foram aplicadas normais climatológicas de precipitação e temperatura do ar referentes ao período de 1961 a 1990. Os totais médios de precipitação (P) foram obtidos da SUDENE (Superintendência de Desenvolvimento do Nordeste) envolvendo 1455 localidades, enquanto que os valores médios mensais de temperatura do $\operatorname{ar}\left(\mathrm{T}_{\mathrm{a}}\right)$ foram obtidos do INMET (Instituto Nacional de Meteorologia), registrados em 75 estações agrometeorológicas convencionais. $\mathrm{Na}$ calibração do método de Thornthaite $(\mathrm{TH})$ para o método de Penman-Monteith (PM) na obtenção da evapotranspiração de referência (ET0), 7 estações automáticas localizadas no Submédio São Francisco foram usadas. Estas últimas estações com observações a cada meia hora de radiação solar global, temperatura e umidade relativa do ar, e velocidade do vento para o cálculo da ET0 estão representadas em azul no lado direito da Figura 1. 


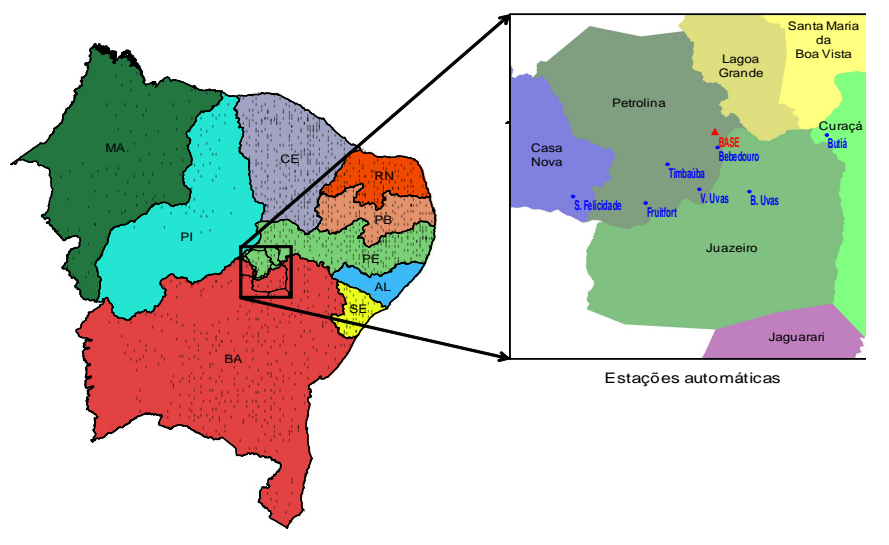

Obs.: As estações convencionais são indicadas por pontos pretos à esquerda e as automáticas por pontos azuis no lado direito

Figura 1. Local ização das estações convencionaisutilizadas na determinação dos índices bioclimáticos da cultura da videira e automáticas para calibrar a evapotranspiração de referência (ETO) obtida pela equação de Thornthwaite (TH ) para o método de Penman-M onteith (PM)

Onde só havia registros de precipitação nas estações convencionais, a temperatura média mensal $\left(\mathrm{T}_{\text {mês }}\right)$ foi estimada em função das coordenadas geográficas de acordo com Cavalcante \& Silva (1994):

$\mathrm{T}_{\text {mês }}=\mathrm{A}_{0}+\mathrm{A}_{1} \lambda+\mathrm{A}_{2} \phi+\mathrm{A}_{3} \mathrm{~h}+\mathrm{A}_{4} \lambda^{2}+\mathrm{A}_{5} \phi^{2}+\mathrm{A}_{6} \mathrm{~h}^{2}+\mathrm{A}_{7} \lambda \phi+\mathrm{A}_{8} \lambda \mathrm{h}+\mathrm{A}_{9} \phi \mathrm{h}$

onde:

$\begin{array}{ll}\lambda & \text { - longitude } \\ \Phi & \text { - latitude } \\ \mathrm{h} & \text { - altitude }\end{array}$

$\mathrm{A}_{0}, \mathrm{~A}_{2}, \ldots, \mathrm{A}_{9}$ - coeficientes de regressão.

Considerando-se que os dados históricos de $\mathrm{T}_{\mathrm{a}}$ são os disponíveis para o cálculo da ET0 em todo o Nordeste brasileiro, o método de Thorntwhwaite (1948) foi primeiramente aplicado para a obtenção dos valores mensais da $\mathrm{ET} 0_{\mathrm{TH}}$ e depois calibrado para o método de Penman-Monteith - ET0 ${ }_{\mathrm{PM}}$ (Allen et al., 1998), compatíveis com resultados dos coeficientes de cultura $(\mathrm{Kc})$ provenientes da videira para mesa, cvs. Itália, Sugraone e para vinho, cv. Syhah (Teixeira et al., 1999; Teixeira et al., 2007), os quais foram obtidos com o método de PenmanMonteith no cálculo da ET0.

De posse dos valores de $\mathrm{T}_{\text {mês }} \mathrm{ET}_{\text {-TH }}$ foi calculado de acordo com as Eqs. 2, 3, 4 e 5:

$$
\mathrm{ET0}_{\mathrm{TH}}=0,5333\left(\frac{10 \mathrm{~T}_{\mathrm{mês}}}{\mathrm{I}_{\text {ano }}}\right)^{\mathrm{a}}\left(\frac{\mathrm{D}_{\text {mês }} \mathrm{N}_{15}}{12}\right)
$$

em que,

$I_{\text {ano }}$ - índice de calor anual obtido pela soma dos índices de calor mensais $\left(\mathrm{i}_{\text {mês }}\right)$ :

$$
\mathrm{i}_{\text {mês }}=\left(\frac{\mathrm{T}_{\text {mês }}}{5}\right)^{1,514}
$$

sendo o expoente a obtido conforme a Eq. 4:

$$
\mathrm{a}=6,7510^{-7} \mathrm{I}^{3}-7,7110^{-5} \mathrm{I}^{2}+1,79 \mathrm{I}+0,49
$$

em que,

$\mathrm{D}_{\text {mês }}$ - número de dias do mês

$\mathrm{N}_{15}$ - fotoperíodo do dia 15 do mês

$\mathrm{N}_{15}$ - é calculado como:

$$
\mathrm{N}_{15}=\frac{2\left[\arccos \left(\operatorname{tg} \Phi \operatorname{tg} \delta_{15}\right)\right]}{15}
$$

em que:

$\delta_{15}$ - declinação do dia 15 do mês

Medições simultâneas da evapotranspiração considerandose condições ótimas de umidade do solo (evapotranspiração potencial - ETp), nas videiras para mesa cvs. Itália e Sugraone, bem como em videira para vinho cv. Syrah, juntamente com estimativas de ET0 pelo método de Penman-Monteith permitiram a obtenção de valores de Kc ao longo das fases fenológicas dos ciclos produtivos (Allen et al., 1998; Teixeira et al., 1999; Teixeira et al., 2007). Neste caso, dados diários de radiação solar global; temperatura e umidade relativa do ar; e velocidade do vento, foram usados para o cálculo dos valores diários de $\mathrm{ET}_{\mathrm{PM}}$.

Três modelos específicos foram desenvolvidos baseados na relação do Kc com os graus-dias acumulados $\left(\mathrm{GD}_{\mathrm{ac}}\right)$ e usados no cálculo da $\mathrm{ET}_{\mathrm{p}}$ após a aplicação de regressões nos valores de $\mathrm{ET} 0_{\mathrm{TH}}$ para equiparar com os valores de $\mathrm{ET} 0_{\mathrm{PM}}$. Os valores de $\mathrm{ET}_{\mathrm{p}}$ para um ciclo produtivo $(\mathrm{CP})$ foram então considerados os requerimentos hídricos das videiras.

Tomando-se valores mensais modelados de GD ac' considerando-se o valor inicial como zero para uma temperatura base de $10{ }^{\circ} \mathrm{C}$ durante os ciclos produtivos (CP), a média dos valores do coeficiente de cultura $\left(\mathrm{Kc}_{\mathrm{CP}}\right)$ foi então multiplicada por $\mathrm{ET}_{\mathrm{CP}}$ :

$$
\mathrm{RHV}_{\mathrm{CP}}=\mathrm{Kc}_{\mathrm{CP}} \mathrm{ET0} 0_{\mathrm{CP}}
$$

em que:

$\mathrm{RHV}_{\mathrm{CP}}$ - requerimento hídrico das videiras para um ciclo produtivo

$\mathrm{Kc}_{\mathrm{CP}}$ - Coeficiente de cultura médio para um ciclo produtivo

$\mathrm{ETO}_{\mathrm{CP}}$ - Evapotranspiração de referência para um ciclo produtivo

Com os valores de $\mathrm{RHV}_{\mathrm{CP}}$ obtidos, o índice termo-hídrico da videira $\left(\mathrm{IHV}_{\mathrm{CP}}\right)$ foi desenvolvido e aplicado na delimitação da aptidão agroclimática da videira no Nordeste brasileiro, variando-se a data de poda.

$$
\mathrm{IHV}_{\mathrm{CP}}=\frac{\mathrm{P}_{\mathrm{CP}}}{\mathrm{RHV}_{\mathrm{CP}}}
$$


em que:

$\mathrm{IHV}_{\mathrm{CP}}$ - índice hídrico da videira para um ciclo produtivo

$\mathrm{P}_{\mathrm{CP}}$ - total de precipitação para um ciclo produtivo

$\mathrm{RHV}_{\mathrm{CP}}$ - requerimento hídrico da videira para um ciclo produtivo

Os dados de temperatura do ar e de precipitação foram interpolados em um ambiente SIG e os modelos foram aplicados às grades com pixels de $0^{\circ} 01$ ' de tamanho. Quatro classes de aptidão foram obtidas através dos valores de $\mathrm{IHV}_{\mathrm{CP}}$, sendo as duas primeiras subdivididas de acordo com os valores de $\mathrm{T}_{\mathrm{CP}}$.

\section{RESULTADOS E DISCUSSÃO}

Como os indicadores bioclimáticos consideram as condições térmicas e hídricas, bem como temperaturas do ar elevadas e excesso de chuvas podem afetar o desenvolvimento das plantas e a qualidade das uvas e do vinho no Nordeste, uma primeira análise de $T_{C P}$ e $P_{C P}$ foi realizada considerando-se os ciclos produtivos de diferentes durações mais frios e mais quentes e os mais chuvosos e mais secos (Figura 2).

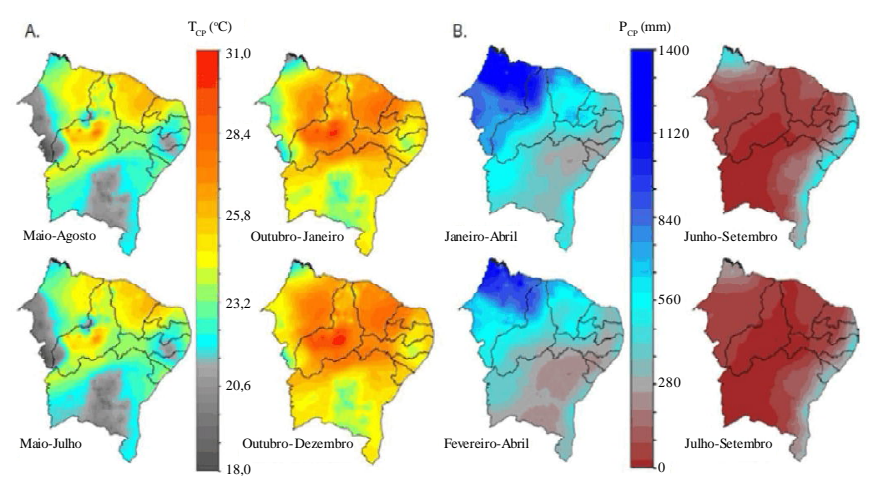

Figura 2. M apeamento das normais climatológicas para ciclos produtivos de três e quatro meses da cultura da videira: $(A)$ temperatura média do ar $\left(T_{C P}\right)$ para ciclos produtivos mais quentes e mais frios; (B) totais médios de precipitação $\left(\mathrm{P}_{\mathrm{CP}}\right)$ para ciclos produtivos mais e menos chuvosos

Considerando-se o Nordeste como um todo, o período de poda com $\mathrm{T}_{\mathrm{CP}}$ de valor mais baixo acontece quando esta é realizada em maio (Figura 2A), para as cultivares de CP tanto de quatro como de três meses de duração. Neste período de ciclo produtivo $(\mathrm{CP})$ que envolve o solistício de inverno no hemisfério Sul, o valor médio de temperatura do ar é em torno de $23^{\circ} \mathrm{C}$. Os valores mais altos de $\mathrm{T}_{\mathrm{CP}}$ são observados para podas em outubro, quando o Sol está na posição zenital na parte central da região. Para os períodos de poda em Maio, muitos pixels apresentam médias para os ciclos produtivos inferiores a $24^{\circ} \mathrm{C}$. Por outro lado percebe-se uma vasta área com valores médios acima de 26 ${ }^{\circ} \mathrm{C}$ quando esta é realizada em outubro, sendo o valor médio neste último mês de $25,5^{\circ} \mathrm{C}$.

Valores de $\mathrm{T}_{\mathrm{CP}}$ elevados são favoráveis para uvas de mesa, pois um elevado teor de açúcar nos frutos é desejável e de acordo com as considerações expostas pela literatura, não há limitações térmicas em nenhum período para o cultivo comercial no Nordeste do Brasil. Por outro lado, para uvas de vinho, embora não havendo limitação térmica para as condições médias, quando a poda é realizada em maio, devido a várias situações que caem dentro do limite de 25 a $30^{\circ} \mathrm{C}$ e acima de 20 ${ }^{\circ} \mathrm{C}$ (Costacurta \& Roselli, 1980; Coombe, 1987), alguma limitação irá ocorrer, quando para podas realizadas em outubro.

Podas no período mais quente poderá proporcionar taxas altas de fluxo de vapor d'água para a atmosfera, contribuindo para elevada produção de biomassa e aumento do conteúdo de açúcar nas uvas. Por outro lado, os vinhos apresentarão altos teores de álcool, com diminuição da acidez total e elevação do $\mathrm{pH}$, podendo apresentar problemas de equilíbrio e conservação, com a presença de taninos imaturos, pela rápida maturação, podendo estar sujeitos a degradações causadas por microorganismos (Ribereau-Gayon et. al, 2004a, b).

Analizando-se os mapas de precipitação (Figura 2B), percebe-se que o período mais chuvoso para videiras de $\mathrm{CP}$ médio de quatro meses ocorre quando a poda é realizada em janeiro, enquanto que para as de três meses este período ocorre com datas de poda em fevereiro. Com relação ao período mais seco também há diferenças, sendo este com podas em junho e em julho, para videiras com CP de quatro e três meses de duração, respectivamente.

No caso das diferenças no regime de precipitação, a duração dos ciclos é mais considerável que no caso do regime térmico, sendo os totais médios para o período mais chuvoso de 593 $\mathrm{mm}$ (janeiro a abril) e para o menos chuvoso de $118 \mathrm{~mm}$ (junho a setembro), respectivamente, para $\mathrm{CP}$ de quatro meses, enquanto que para as variedades com três meses de duração do ciclo produtivo estes totais são de $464 \mathrm{~mm}$ (fevereiro a abril) e de $74 \mathrm{~mm}$ (julho a setembro). Percebe-se, portanto menores riscos causados por excesso de chuvas ao longo do ano para a videira com menor $\mathrm{CP}$.

Durante os períodos mais chuvosos, o Estado do Maranhão e o lado noroeste do Estado do Piaú são os mais problemáticos com relação a excesso hídrico para as videiras, principalmente para aquelas com $\mathrm{CP}$ de quatro meses, devido às influências do clima amazônico, com totais de precipitação acima de 800 $\mathrm{mm}$ por CP, para podas de janeiro a abril. Quando estas ocorrem entre junho e setembro, quase toda a região apresenta valores de $\mathrm{P}_{\mathrm{CP}}$ inferiores a $400 \mathrm{~mm}$ por $\mathrm{CP}$.

A Figura 3 apresenta os resultados das equações de regressão para calibração da $\mathrm{ET} 0_{\mathrm{TH}} \mathrm{em} \mathrm{ET0} 0_{\mathrm{PM}}$ antes da aplicação do modelo que relaciona $\mathrm{Kc}$ com $\mathrm{GD}_{\mathrm{ac}}$ em escala regional, para a obtenção dos requerimentos hídricos das videiras de acordo com a época de poda no Nordeste do Brasil.

Considerando-se todos os meses do ano, a correlação entre os métodos foi baixa (Figura 4A), então as equações específicas para o primeiro (Figura 4B) e segundo (Figura 4C) semestres do ano foram aplicadas separadamente.

Uma melhor distribuição em torno da linha 1:1 ocorreu no primeiro semestre do ano (Figura 4B), enquanto que no segundo semestre há uma grande sub estimativa do método TH com relação aos resultados pelo método PM (Figura 4C). Esta sub-estimativa pode ser explicada pelo fato do primeiro método considerar apenas a temperatura do ar e as coordenadas 
A.

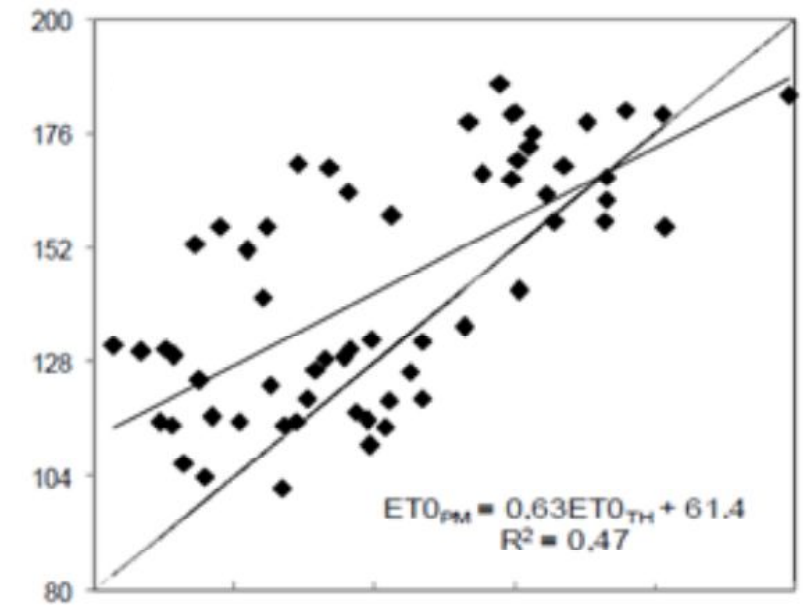

B.

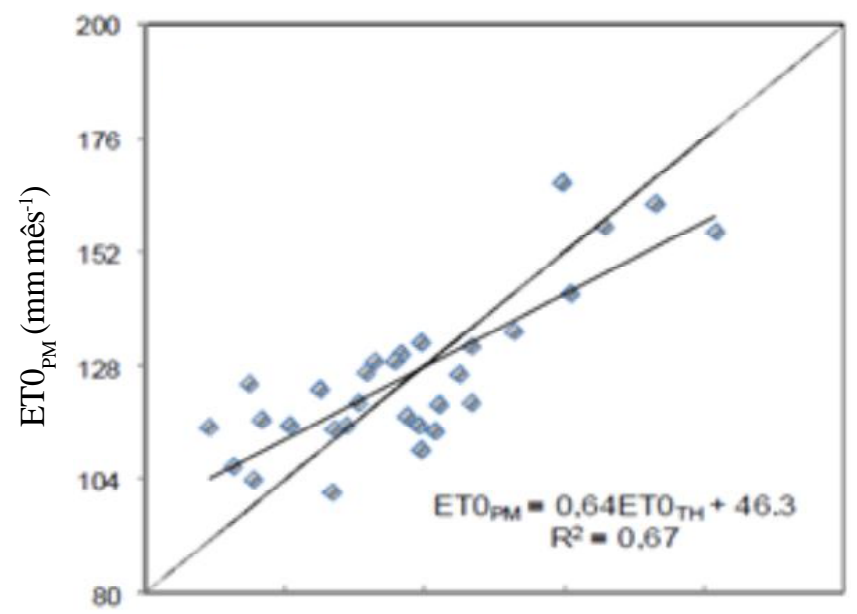

C.

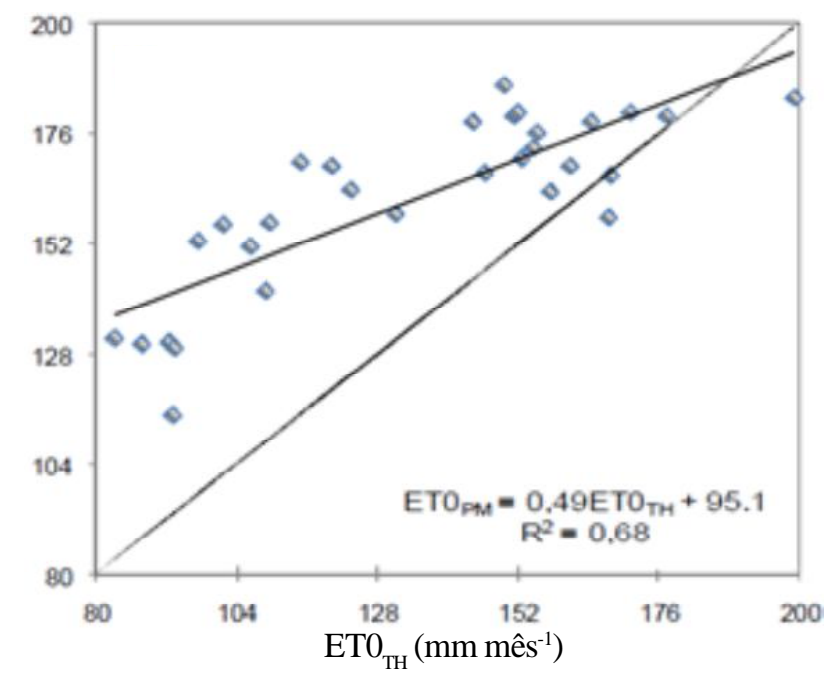

Figura 3. Relações entre os valores mensais da evapotranspiração de referência cal culada pelo método de Penman-M onteith $\left(E T O_{P M}\right)$, e pelo método de Thorntwaite $\left(E T 0_{T H}\right)$. (A) envolvendo todos os meses do ano; (B) envolvendo apenas os meses do primeiro semestre; $(C)$ envolvendo apenas os meses do segundo semestre

geográgficas no cálculo da $\mathrm{ET}_{\mathrm{TH}}$, enquanto que para a obtenção de $\mathrm{ET} 0_{\mathrm{PM}}$, os outros parâmetros agrometeorológicas de entrada são radiação solar global, umidade relativa do ar e
A.

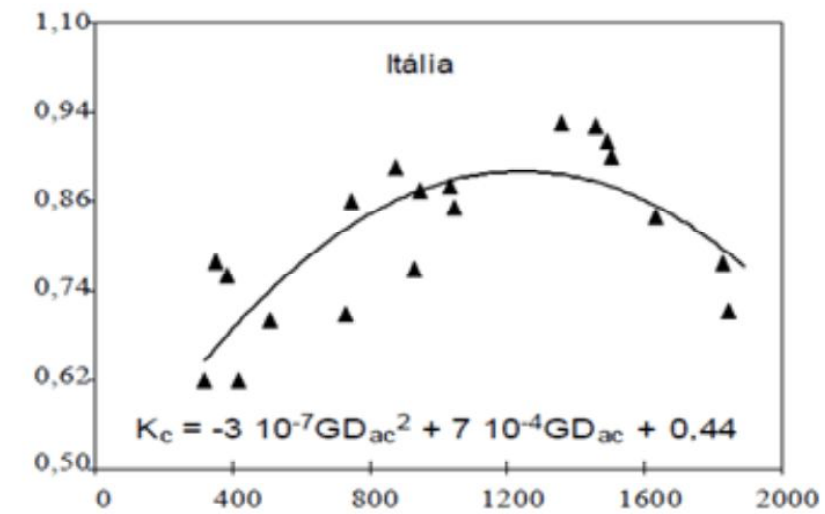

B.

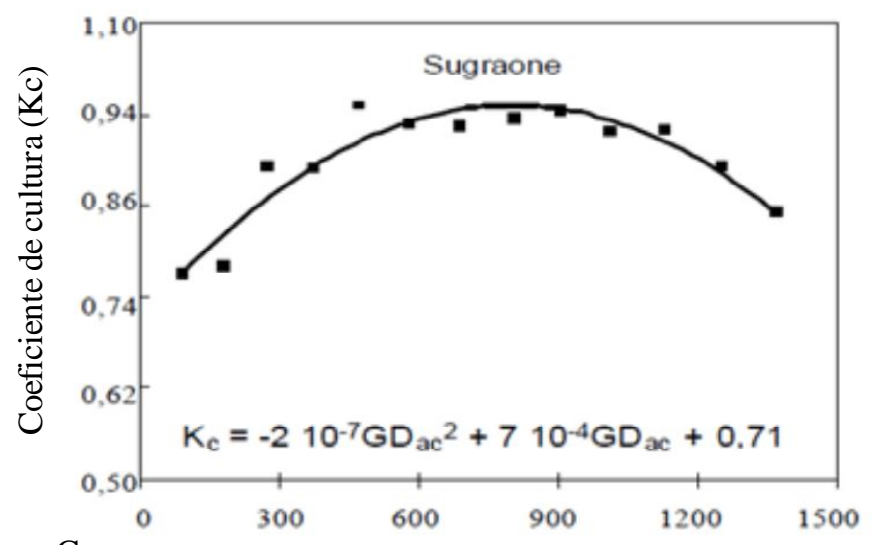

C.

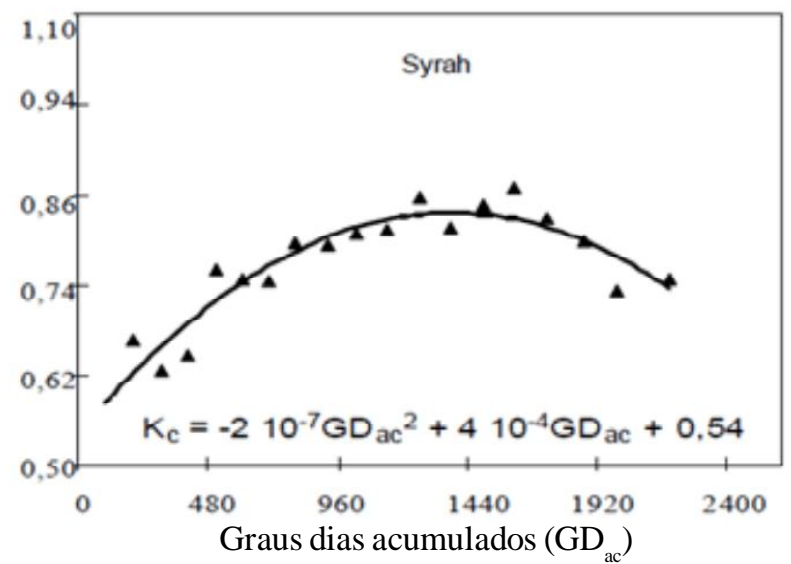

Obs.: Os triângulos representam ciclos de 4 meses enquanto que os quadrados representam ciclos de 3 meses

Figura 4. Variação dos valores semanais do coeficiente de cultura $(\mathrm{Kc})$ em função dos graus dias acumulados (GD ac). (A) videiras para mesa com sementes (cv. Itália),

(B) videiras para mesa sem sementes (cv. Sugraone) e (C) videiras para vinho (cv. Syrah). Considerou-se a temperatura basal de $10^{\circ} \mathrm{C}$, na região semiárida do $\mathrm{N}$ ordeste do Brasil

velocidade do vento. Para o segundo semestre, a omissão destes últimos parâmetros proporciona um maior efeito nos resultados para as condições climáticas do Nordeste brasileiro.

Após a calibração de de $\mathrm{ET}_{\mathrm{TH}}$ em ET0 $_{\mathrm{PM}}$ os resultados foram usados com os valores de Kc modelados em função de $\mathrm{GD}_{\text {ac }}$ para as videiras para mesa, cvs. Itália e Sugraone e para a videira para vinho cv. Syrah. Os resultados das equações de regressão são apresentados na Figura 4, as quais foram 
aplicadas às grades de $\mathrm{GD}_{\mathrm{ac}}$ para a obtenção dos valores regionais de $\mathrm{RHV}_{\mathrm{CP}}$.

Os valores de entrada representados na Figura 4 resultaram de medições paralelas da evapotranspiração dos parreirais em ótimas condições de umidade (ETp) e da ET0 na vizinhança dos experimentos de balanço de energia (Teixeira et al., 1999; Teixeira et al., 2007).

No trabalho atual os modelos foram desenvolvidos e as equações foram aplicadas aos pixels de $\mathrm{GD}_{\mathrm{ac}}$ para a extrapolação dos requerimentos hídricos dos parreirais, aqui representado pela ETp. Para uvas de mesa sem sementes, foi tomada como referência a variedade Itália com quatro meses de duração média do $\mathrm{CP}$, enquanto que para aquelas sem sementes a variedade Sugraone foi considerada representativa, com três meses de duração média do CP. Com relação a uvas para vinho, a variedade Syrah foi considerada como representativa, geralmente com quatro meses de duração do CP na região.

Os modelos representados na Figura 4 têm a vantagem de incorporarem o efeito da temperatura do ar no comprimento dos estágios das videiras e da extrapolação dos valores de Kc para diferentes condições térmicas, sendo também úteis para a quantificação dos efeitos das mudanças climáticas nos requerimentos hídricos, para cenários futuros.

Aplicou-se a Eq. 7, considerando-se a duração e as condições térmicas dos ciclos produtivos sob podas em diferentes épocas do ano, para a classificação das regiões com maior ou menor grau de aptidão agroclimática. Como a temperatura do ar e as condições hídricas afetam diferentemente as videiras para mesa e para vinho, os critérios de delimitação agroclimáticos usados entre as duas culturas foram diferentes.

Para as videiras de mesa, elevados teores de açúcar são desejáveis e estes são atingidos sob altos valores de temperatura do ar (Keller, 2010). Como os valores de $T_{C P}$ estão dentro dos limites registrados na região Nordeste (ver Figura 2A) conclui-se que as áreas mais quentes são as favoráveis, enquanto que o excesso hídrico em algumas regiões e épocas de poda vai afetar a qualidade e produtividade das uvas de mesa mais do que em videiras para vinho (Teixeira et al., 2007).

Consideraram-se então duas faixas (classes C1 e C2) de melhor aptidão para produção de uvas de mesa, com valores de $\mathrm{IHV}_{\mathrm{CP}}$ menores ou iguais a 1,5, de acordo com o valor limite de $\mathrm{T}_{\mathrm{CP}}$ de $24^{\circ} \mathrm{C}$, o qual é intermediário na escala da Figura $2 \mathrm{~A}$. Uma classe subsequente $(\mathrm{C} 3)$ de menor aptidão com $\mathrm{IHV}_{\mathrm{CP}}$ maior do que 1,5 e menor ou igual a 3,5 , onde na medida em que o grau de umidade natural se eleva, a produção e a qualidade das uvas seria afetada. Por fim, uma última classe foi considerada (C4), para as localidades com $\mathrm{IHV}_{\mathrm{CP}}$ superior a 3,5, classificadas como as de menor aptidão agroclimática para a produção comercial de uvas de mesa.

A vantagem desta classificação com relação a Teixeira et al. (2002) para o Estado da Bahia, é que a atual considera os requerimentos hídricos específicos na escala temporal mensal, baseados em resultados experimentais em parreirais e a consideração da variação da época de poda ao longo do ano em todo o Nordeste brasileiro, envolvendo ainda as variedades com diferentes durações nos ciclos produtivos.

A Figura 5 apresenta os resultados da classificação agroclimática da variedade para mesa Itália, representativa da videira com sementes com CP médio de quatro meses de duração, considerando-se podas em diferentes meses do ano.
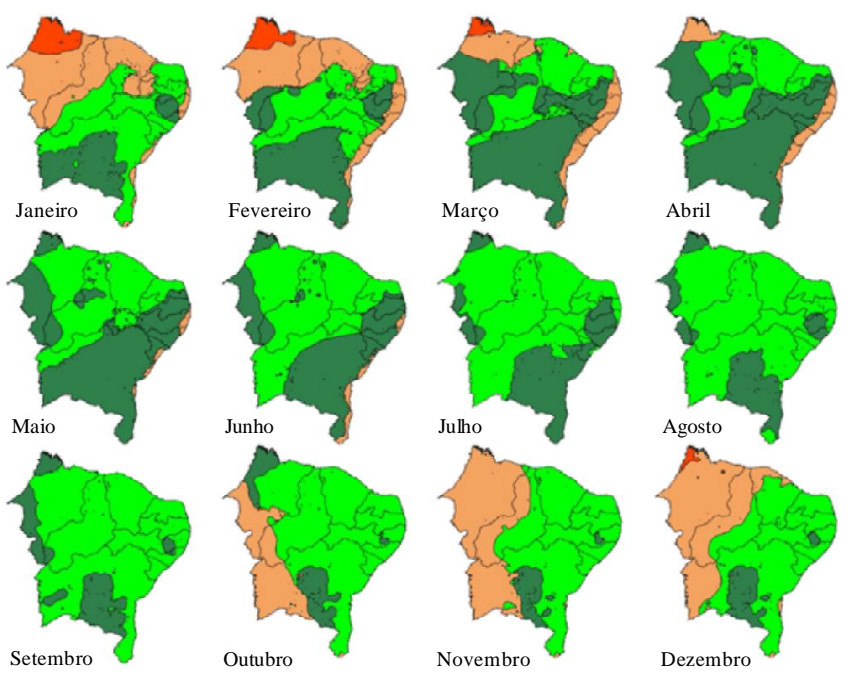

$\mathrm{Cl}: \mathrm{HHV}_{\mathrm{CP}} \leq 1,5 \mathrm{e} \mathrm{T}_{\mathrm{CP}} \geq 24 \mathrm{C}$

$\mathrm{C} 2: \mathrm{IHV}_{\mathrm{CP}} \leq 1.5 \mathrm{eT}<24^{\circ} \mathrm{C}$

$\mathrm{C} 3: 1,5<\mathrm{HHV}_{\mathrm{Cp}} \leq 3,5$

$\mathrm{C} 4: \mathrm{IHV}_{\mathrm{cp}}>3,5$

Figura 5. D elimitação dos graus de aptidão agroclimática para videira de mesa cv. Itália de acordo com as épocas de poda no Nordeste do Brasil

Os períodos com maior aptidão climática (classes C1 e C2) para a variedade Itália são aqueles quando a poda é realizada de julho a setembro, com o $\mathrm{IHV}_{\mathrm{CP}}$ chegando a valores em torno de 0,3 em julho e a $\mathrm{T}_{\mathrm{CP}}$ acima de $24{ }^{\circ} \mathrm{C}$ durante o período, destacando-se como classe $\mathrm{C} 1$ as áreas ao oeste dos estados da Bahia (BA), Pernambuco (PE), Paraíba (PB) e quase todas as áreas dos estados do Rio Grande do Norte (RN), Ceará (CE), Piauí (PI) e Maranhão (MA). A classe de pior aptidão agroclimática (C4) ocorre apenas no Estado do Maranhão (MA) quando a poda é realizada de dezembro a março, principalmente quando esta ocorre em janeiro.

Para videiras de mesa, com CP médio de três meses de duração, as sem sementes, as mesmas classes foram consideradas com relação às variedades com sementes, entretanto, como a duração do ciclo produtivo é menor, podese fazer um escalonamento com menores possibilidades de danos causados pelo excesso hídrico. As classes de aptidão agroclimática para este caso são apresentadas na Figura 6 .

A melhor época de poda das videiras para mesa sem sementes é deslocada para o período de agosto a setembro, com relação às variedades com sementes, de $\mathrm{CP}$ médio de quatro meses, ocorrendo uma redução no valor médio de $\mathrm{IHV}_{\mathrm{CP}}$ para 0,2 com podas no mês de agosto, e valores de $\mathrm{T}_{\mathrm{CP}}$ também acima de $24^{\circ} \mathrm{C}$. Uma maior abrangência de áreas $\mathrm{C} 1$ é verificada e a classe $\mathrm{C} 4$ neste caso aparece em apenas pequenas áreas com podas em janeiro e fevereiro no noroeste Maranhão, sendo que mesmo neste estado com influência do clima amazônico, uma grande parte de áreas sendo classificada como $\mathrm{C} 1$, ocorre para períodos de poda de julho a setembro. 

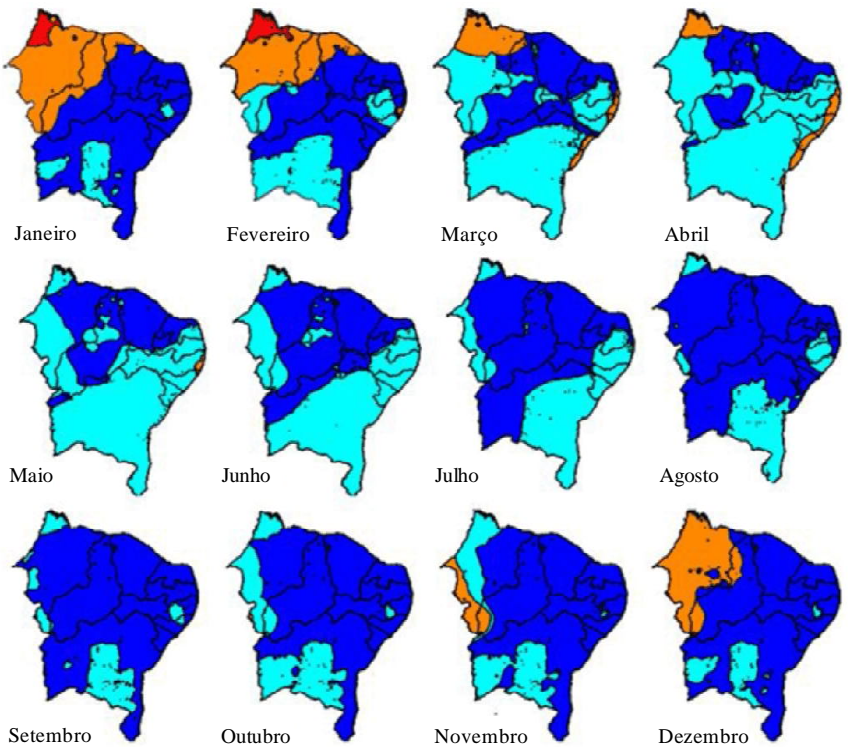

$\mathrm{Cl}: \mathrm{HVV}_{\mathrm{CP}} \leq 1,5 \mathrm{e} \mathrm{T}_{\mathrm{CP}} \geq 24 \mathrm{C}$

$\mathrm{C} 2: \mathrm{HHV}_{\mathrm{CP}} \leq 1,5 \mathrm{e} \mathrm{T}_{\mathrm{CP}}<24 \mathrm{C}$

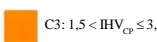

$\mathrm{C} 4: \mathrm{IHV}_{\mathrm{CP}}>1,5$

Figura 6. D elimitação dos graus de aptidão agroclimática para videira de mesa cv. Sugraone de acordo com asépocas de poda no Nordeste do Brasil

Tanto para videiras de mesa com sementes como sem sementes, percebe-se uma grande faixa de aptidão C1 e C2 para podas entre julho a setembro. As áreas classificadas como agroclimaticamente mais aptas em ambos os tipos de videira analisados apresentam maiores disponibilidades térmicas e baixos índices de umidade climatológica, proporcionando menor ocorrência de doenças bem como redução dos efeitos do excesso de chuvas sobre o tamanho e qualidade das uvas, tendo, portanto maior potencial climático para a produção de uvas de mesa em condições de irrigação. Entretanto, cautela deve ser tomada com relação ao manejo racional dos recursos hídricos, pois condições térmicas elevadas proporcionam uma maior produção, mas acarretam em uma maior taxa evaporativa, devendo-se considerar a possibilidade de conflitos no uso da água no futuro, na escolha das áreas para exploração comercial.

Com relação à videira para vinho, os mesmos indicadores foram usados, porém com critérios diferentes na classificação agroclimática. No aspecto térmico, temperaturas elevadas irão causar altos níveis de álcool, baixa acidez e elevado pH nos vinhos, os quais são indesejáveis comercialmente (Ollat et al., 2002; Ribereau-Gayon et al., 2004a, b; Orduña, 2010). Do ponto de vista hídrico o excesso de chuvas é menos problemático que no caso de videira para mesa, sendo então considerado um limite do $\mathrm{IHV}_{\mathrm{CP}}$ ligeiramente superior para o zoneamento baseado em observações de campo (Teixeira et al., 2007; Teixeira, 2009).

Quatro classes de aptidão agroclimática, como no caso da videira para mesa, foram também consideradas e apresentadas na Figura 7, porém neste caso para a elaboração comercial de vinhos típicos de regiões tropicais.

Duas classes de melhor aptidão sob condições de irrigação foram consideradas com $\mathrm{IHV}_{\mathrm{CP}}$ menor que 2 subdivididas de acordo com o valor de $\mathrm{T}_{\mathrm{CP}}$ limite de $24,0^{\circ} \mathrm{C}$. Embora estas classes apresentem condições de umidade climatológica do ar ideal,
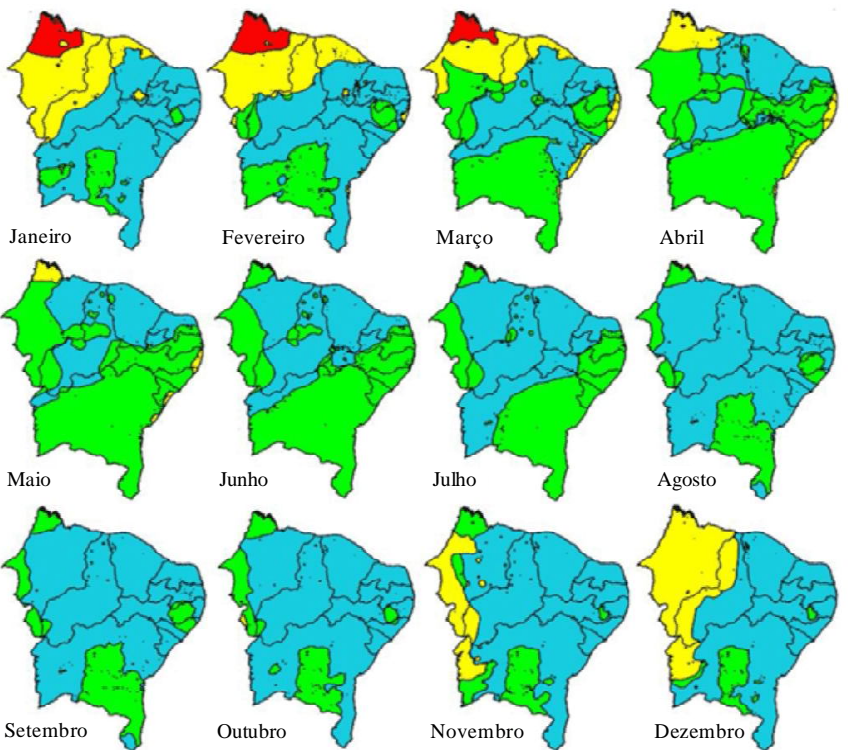

$\mathrm{Cl}: \mathrm{HH}_{\mathrm{CP}} \leq 2 \mathrm{e} \mathrm{T}_{\mathrm{Cp}}<24^{\circ} \mathrm{C}$
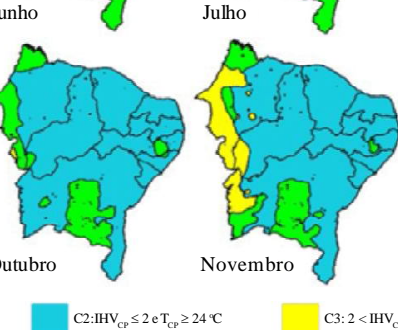

Agosto

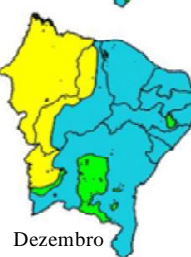

Figura 7. D elimitação dos graus de aptidão agroclimática para videira de vinho cv. Syrah de acordo com as épocas de poda no Nordeste do Brasil

valores de $T_{C P}$ maior ou igual a este limite poderiam trazer algum desequilíbrio e instabilidade nos vinhos produzidos, com elevados níveis de álcool, baixa acidez, altos valores de $\mathrm{pH}$, e instabilidade nos componentes aromáticos e fenólicos, demandando mais cuidado na manutenção da qualidade e tipicidade do vinho nestas condições (Ribereau-Gayon et al., 2004a, b).

Com relação às condições de umidade natural, foi ainda considerada uma classe de menor aptidão com valores de $\mathrm{IHV}_{\mathrm{CP}}$ maiores que 2 e menores ou igual a 4 , onde na medida em que o grau de umidade aumenta, a qualidade do vinho produzido pode ser afetada. As áreas com datas de poda dentro da pior classe de aptidão foram consideradas aquelas que apresentam valores de $\mathrm{IHV}_{\mathrm{CP}}$ maiores que 4, quando teriam grandes problemas para a produção das uvas para vinho devido aos altos valores de umidade que comprometem a sanidade das uvas, e, consequentemente, o potencial enológico. O efeito desta condição hídrica em conjunto com valores altos de temperatura do ar compromete mais ainda a qualidade final dos vinhos elaborados.

Considerando a região Nordeste como um todo, o período com melhor aptidão climática das videiras para vinho (classes C1 e C2) é quando a poda é realizada em junho, situação em que os valores de $\mathrm{T}_{\mathrm{CP}}$ e do $\mathrm{IHV}_{\mathrm{CP}}$ são, respectivamente, $0,50 \mathrm{e}$ $22,9{ }^{\circ} \mathrm{C}$. A pior classe de aptidão (classe $\mathrm{C} 4$ ), ocorre quando as podas são realizadas entre janeiro e março, apenas no lado noroeste do Estado do Maranhão (MA). Torna-se importante observar, que mesmo as áreas dentro desta classificação não sendo indicadas para este período, elas apresentam boa aptidão climática (classes C1 e C2) quando as datas de poda ocorrem de junho a outubro.

Para otimização do cultivo da videira para vinho, antes dos períodos de poda de melhor aptidão, a cultura poderia ser deixada em repouso e entre linhas cultivadas com gramíneas e leguminosas 
nas condições chuvosas. Após este período, e antes do ciclo produtivo, estas espécies poderiam ser incorporadas no solo como matéria orgânica. Destaques para as regiões mais aptas para a elaboração de vinhos típicos são para os Estados da Bahia (BA), Pernambuco (PE), Paraíba (PB), Alagoas (AL), e Sergipe (SE), e ainda a parte sudoeste do Estado do Maranhão (MA).

As áreas classificadas como mais aptas para o cultivo comercial, tanto para as videiras para mesa como para vinho, apresentam baixos níveis de umidade climática, promovendo uma menor incidência de doenças, bem como redução dos problemas causados diretamente às videiras pelo excesso de precipitação, permitindo a obtenção de uvas de melhor qualidade e vinhos típicos. Entretanto, para programas expansão destas culturas, estas áreas devem ser delimitadas ainda de acordo com outras características ambientais, como solos, enxertos, adaptação de cultivares, para se utilizar todo o potencial do Nordeste do Brasil para a produção comercial de uvas para mesa e vinhos tropicais.

\section{Conclusões}

1. Modelos simples, baseados no coeficiente de cultura, na evapotranspiração de referência, e nos graus-dias acumulados, juntamente com dados históricos de precipitação e de temperatura do ar permitiram o desenvolvimento de índices bioclimáticos da videira para a delimitação de zonas com diferentes aptidões agroclimáticas.

2. As melhores opções para produção comercial de uvas para mesa foram para oeste dos estados da Bahia, Pernambuco, Paraíba e quase todas as áreas dos estados do Rio Grande do Norte, Ceará, Piauí e Maranhão com podas realizadas no período julho a setembro.

3. As melhores opções para a elaboração comercial de vinhos típicos, foram para os estados Bahia, Pernambuco, Paraíba, Alagoas e Sergipe e a parte oeste do Estado do Maranhão para datas de poda entre maio e junho.

4. Diferentes critérios entre videiras para mesa e para vinho devem ser tomados para a delimitação de zonas de aptidão climática.

\section{AgRAdeCimentos}

Os autores são gratos à FACEPE (Fundação de Ciência e Tecnologia do Estado de Pernambuco) pelo financiamento do projeto sobre produtividade da água em situações de rápida mudança de uso da terra, o qual permitiu a aplicação de dados climáticos provenientes de estações agrometeorológicas automáticas.

\section{LITERATURA CITADA}

Allen, R. G.; Pereira, L. S.; Raes, D.; Smith, M. Crop evapotranspiration. Guidelines for computing crop water requirements. Rome: FAO, 1998. 300p. Irrigation and Drainage Paper, 56
Azevedo, P. V. de; Soares, J. M.; Silva, V. P. R. da; Silva, B. B. da; Nascimento, T. Evapotranspiration of "Superior" grapevines under intermittent irrigation. Agricultural Water Management, v.95, p.301-308, 2007.

Cavalcanti, E. P.; Silva, E. D. V. Estimativa da temperatura do ar em função das coordenadas locais. In: Congresso Brasileiro de Meteorologia, 8, e Congresso Latino-Americano e Ibérico de Meteorologia, Belo Horizonte, 1994, Belo Horizonte, Anais... Minas Gerais: SBMET, 1994. p.154-157.

Coombe, B. G. Influence of temperature on composition and quality of grapes. Acta Horticulturae, v.206, p.23-35, 1987.

Costacurta, A.; Roselli, G. Critères climatiques et edaphiques pour l'établissement des vignobles. Bulletin de L'O.I.V, v.53, p.783-786, 1980.

Keller, M. Managing grapevines to optimize fruit development in a challenging environment: A climate change primer for viticulturists. Australian Journal of Grape and Wine Research, v.16, p.56-69, 2010.

Moller, M.; Alchanatis, V.; Cohen, Y.; Meron, M.; Tsipris, J.; Naor, A.; Ostrovsky, V.; Sprintsin, M.; Cohen, S. Use of thermal and visible imagery for estimating crop water status of irrigated grapevine. Journal of Experimental Botany, v.58, p.827-838, 2007.

Ollat, N.; Diakou-Verdin, P.; Carde, J. P.; Barrieu, F.; Gaudillère, J. P.; Moing, A. Grape berry development: a review. Journal International des Sciences de la Vigne et du Vin, v.36, p.109131, 2002.

Orduña, R. M. de. Climate change associated effects on grape and wine quality and production. Food Research International, Davis, USA: Acta Horticulturae, v.43, p.1844$1845,2010$.

Ortega-Farias, S.; Carrasco, M.; Olioso, A.; Acevedo, C.; Poblete, C. Latent heat flux over cabernet sauvignon vineyard using the Shuttleworth and Wallace model. Irrigation Science, v.25, p.161-170, 2007.

Rana, G.; Katerji, N.; Michele, I. M.; Hammami, A. Microclimate and plant water relationship of the "overhead" table grape vineyard managed with three different covering techniques. Scientia Horticulturae, v.102, p.105-120, 2004.

Ribereau-Gayon, P.; Dubourdieu, D.; Donèche, B.; Lonvaud, A. Tratité d'Oenologie. Tome 1: Microbiologie du Vin. Vinifications, 5.ed. Dunod Paris, 2004a. 661p.

Ribereau-Gayon, P.; Glories, Y.; Maujean, A.; Dubourdieau, D. Tratité d'Oenologie. Tome 2: Chimie du vin. Stabilisation et traitements, 5.ed., Dunod Paris, 2004b. 566p.

Tarara, J. M.; Lee, J.; Spayd, S. E.; Scajel, C. F. Berry temperature and solar radiation alter acylation, proportion, and concentration of anthocyanin in merlot grapes. American Journal of Enology and Viticulture, v.59, p.235-247, 2008.

Teixeira, A. H. de C. Water productivity assessments from field to large scale: a case study in the Brazilian semi-arid region. Saarbrücken, Germany: LAP Lambert Academic Publishing, 2009, 226p.

Teixeira, A. H. de C.; Azevedo, P. V. de; Silva, B. B. da; Soares, J. M. Consumo hídrico e coeficiente de cultura da videira na região de Petrolina, PE. Revista Brasileira de Engenharia Agrícola e Ambiental, v.3, p.413-416, 1999. 
Teixeira, A. H. de C.; Bastiaanssen, W. G. M.; Ahmad, M. D.; Bos, M. G. Reviewing SEBAL input parameters for assessing evapotranspiration and water productivity for the LowMiddle São Francisco River basin, Brazil Part A: Calibration and validation. Agricultural and Forest Meteorology, v.149, p.462-476, 2009a.

Teixeira, A. H. de C.; Bastiaanssen, W. G. M.; Ahmad, M. D.; Bos, M. G. Reviewing SEBAL input parameters for assessing evapotranspiration and water productivity for the LowMiddle São Francisco River basin, Brazil Part B: Application to the large scale. Agricultural and Forest Meteorology, v. 149, p. $477-490,2009$ b.

Teixeira, A. H. de C.; Bastiaanssen, W. G. M.; Ahmad, M. D.; Bos, M. G. Analysis of energy fluxes and vegetation-atmosphere parameters in irrigated and natural ecosystems of semi-arid Brazil. Journal of Hydrology, v.362, p.110-127, 2008.

Teixeira, A. H. de C.; Bastiaanssen, W. G. M.; Bassoi, L. H. Crop water parameters of irrigated wine and table grapes to support water productivity analysis in Sao Francisco River basin, Brazil. Agricultural Water Management, v.94, p.31-42, 2007.
Teixeira, A. H. C.; Souza, R. A.; Ribeiro, P. H. B.; Reis, V. C. S.; Santos, M. G. L. Aptidão agroclimática da cultura da videira no Estado da Bahia, Brasil. Revista Brasileira de Engenharia Agrícola e Ambiental, v.6, p.107-111, 2002.

Thornthwate, C. W. An approach toward a rational classification of climate. Geographical Review, v. 38, p.55-94, 1948.

Williams, L. E.; Ayars, J. E. Grapevine water use and the crop coefficient are linear functions of the shaded area measured beneath the canopy. Agricultural and Forest Meteorology, v.132, p.201-211, 2005.

Williams, L. E.; Phene, C. J.; Grimes, D. W.; Trout, T. J. Water use of mature Thompson Seedless grapevines in California. Irrigation Science, v.22, p.11-18, 2003.

Yunusa, I. A. M.; Walker, R. R.; Lu, P. Evapotranspiration components from energy balance, sapflow and microlysimetry techniques for an irrigated vineyard in inland Australia. Agricultural and Forest Meteorology, v.127, p.93107, 2004. 